\title{
极性聚合物粘结剂的结构和物性对锂离子电池的影响
}

\author{
郭容男，韩伟强 \\ (浙江大学 材料科学与工程学院, 杭州 310027)
}

摘 要: 粘结剂在锂离子电池中虽用量少, 但是对锂离子电池的性能有较大影响。传统粘结剂聚偏氟乙烯与活性物 质间形成的范德华力较弱, 不能满足现代锂离子电池, 特别是高比容量锂离子电池的要求。大部分电极材料表面具 有极性基团, 这些基团可与极性聚合物间形成较强的作用力, 故极性聚合物粘结剂成为当前的研究热点。极性聚合 物粘结剂对锂离子电池的影响与诸多因素有关。本文主要讨论了聚合物粘结剂的结构和物性对锂离子电池性能的 影响, 包括聚合物的结构特征、粘结性、力学性能和导电性等因素, 进而从分子层次提出了设计下一代粘结剂的方 法, 并展望了粘结剂的未来发展方向。

关 键 词: 极性聚合物; 粘结剂; 锂离子电池; 综述

中图分类号: TQ174 文献标识码: A

\section{Effects of Structure and Properties of Polar Polymeric Binders on Lithium-ion Batteries}

\author{
GUO Rong-Nan, HAN Wei-Qiang \\ (School of Materials Science and Engineering, Zhejiang University, Hangzhou 310027, China)
}

\begin{abstract}
Binders have a great impact on the performance of lithium-ion batteries, although small doses of binder is applied. The traditional binder poly (vinylidene fluoride) cannot meet the requirements of modern lithium-ion batteries, especially those with high specific capacity, because it interacts with electrode materials through weak Van de Waals force. The surfaces of most electrode materials have polar groups which can strongly interact with polar polymeric binders. Therefore, the polar polymeric binders have been paid much attention, recently. Many factors of polar polymeric binders influence the properties of lithium-ion batteries. This review mainly focuses on the impacts of structure properties of polar polymeric binders on lithium-ion batteries, including structural feature, adhesiveness, mechanical properties, conductivity, and other properties Besides, we propose the strategies of designing next-generation binders for lithium-ion batteries from molecular level, and claim the future direction and prospects of the binders.
\end{abstract}

Key words: polar polymer; binder; lithium-ion battery; review

科学技术的快速发展一方面消耗了大量不可再 生化石燃料, 另一方面造成了严重的环境污染, 威 胁人类的健康。为了世界的可持续发展和人类的生 存环境, 急需开发洁净、安全和可再生的新能源。 锂离子电池(LIBs) 因具有能量密度高、寿命长、倍 率性能好和绿色环保等特点而备受关注。目前 LIBs 已广泛应用于便携式电池产品, 但是应用于动力汽
车时, 其能量密度和循环性能仍需要进一步提高。 对此，开发新型高比容量活性物质或改变活性物质 的形貌可提高电极的比容量和循环稳定性 ${ }^{[1-4]}$, 但 是用量较少的粘结剂的作用也不可忽视。粘结剂的 主要作用是将活性物质、导电剂和集流体粘结在一 起，保证电极的完整性。不同粘结剂对电极的初始 比容量、库伦效率和电化学循环稳定性的影响有较

收稿日期：2019-01-24; 收到修改稿日期：2019-05-07

作者简介: 郭容男(1987-), 女, 博士研究生. E-mail: 11626022@zju.edu.cn

通讯作者: 韩伟强, 教授. E-mail: hanwq@zju.edu.cn 
大差异 ${ }^{[5-8]}$ 。传统商用粘结剂聚偏氟乙烯(PVDF)由 于成本高、与活性物质的粘结力差和使用有毒溶剂 $N$-甲基吡咯烷酮等缺点已不能满足开发高比容量电 极和环保的要求 ${ }^{[9-10]}$ 。而低成本且环保的羧甲基纤 维素钠 $(\mathrm{CMC})$ 尽管可以显著提高粘结性能和电极的 初始库伦效率, 但是该粘结剂的柔性差, 不能承受 高比容量活性物质的体积膨胀 ${ }^{[11-12]}$, 所以目前极性 聚合物仍是 LIBs 的主要粘结剂。为了便于学者们更 好地设计开发新型高效粘结剂, 本文论述了极性聚 合物的结构、粘结性、力学性能和导电性等因素对 LIBs 性能的影响(图 1), 并展望了未来 LIBs 用粘结 剂的发展方向。

\section{1 极性聚合物的结构特征}

聚合物是由大量的结构单元按照不同的键合方 式连接而成, 故不同聚合物的结构特征不同, 对 LIBs 电化学性能的影响也有所差异。下面将具体论 述极性聚合物的结构对 LIBs 电化学性能的影响。

\section{1 结构单元}

结构单元按极性分为极性和非极性结构单元。 极性结构单元可与表面含极性基团的被粘物质形成 较强的作用力 ${ }^{[13]}$ 。如含羧基、羟基和异氰酸酯基团 等极性基团的聚合物在一定条件下与被粘物形成化 学键, 提高粘结强度和对活性物质的分散性 ${ }^{[14-15]}$ 。 大部分活性物质表面呈极性, 故极性聚合物粘结剂 具有更好的粘结性能。极性基团的种类及数量对电 极材料的粘结性的影响不同。如 $\mathrm{Si}$ 表面含有极性基 团 $\mathrm{SiO}_{x}$ 及 $-\mathrm{OH}$, 以含有极性结构单元的 $\mathrm{CMC}$ 为粘 结剂, 则 $\mathrm{CMC}$ 中大量 $-\mathrm{OH}$ 和- $\mathrm{COO}^{-}$极性基团可与
活性物质表面的 $-\mathrm{OH}$ 或 $\mathrm{SiO}_{x}$ 形成共价键和(或)氢键 (图 2), 故对 $\mathrm{Si}$ 的粘结性极高, 极大提高了电极的比 容量和电化学循环稳定性 ${ }^{[16]}$ 。含有其他极性结构单 元的极性聚合物对 LIBs 的电化学性能影响如表 1 所 示。此外极性结构单元可提高 $\mathrm{Si}$ 颗粒的分散性 ${ }^{[27-28]}$ 。 同时含不同极性基团的聚合物也可通过极性基团间 的协同作用提高粘结力。如 $\mathrm{CMC}$ 链上引入聚丙烯 酸钠(NaPAA-g-CMC)、聚丙烯酸(PAA)或海藻酸钠 (SA)中引入支链多巴胺(PAA-C 或 Alg-C, 图 3), 均 可极大提高聚合物的粘结性能和硅负极的电化学循 环稳定性 ${ }^{[29-30]}$ 。Alg-C 粘结剂可使硅负极在电流密 度为 $2100 \mathrm{~mA} / \mathrm{g}$ 的条件下循环 400 圈后, 比容量仍 高于 $2000 \mathrm{mAh} / \mathrm{g}^{[29]}$ 。但是对于嵌锂时体积膨胀巨大 的活性物质，如果极性结构单元含量过大，由于极 性单元间作用力如氢键阻碍分子链的自由旋转，聚 合物柔性过差，不利于承受活性物质体积膨胀产

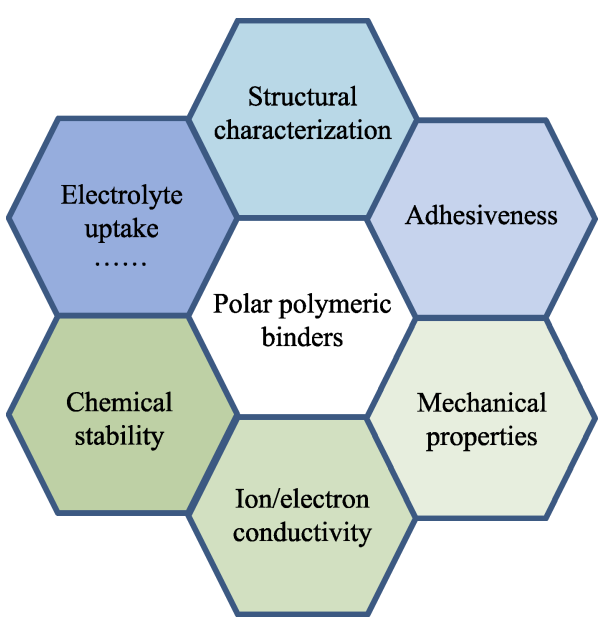

图 1 极性聚合物粘结剂对 LIBs 性能的影响因素

Fig. 1 Influences of polar polymeric binders on properties of LIBs

表 1 极性聚合物粘结剂在 LIBs 中的应用

Table 1 Applications of polar polymeric binders in LIBs

\begin{tabular}{|c|c|c|c|c|}
\hline Binder & Application & Adhesion & Specific capacity $/\left(\mathrm{mAh} \cdot \mathrm{g}^{-1}\right)$ & Ref. \\
\hline \multicolumn{5}{|c|}{ Linear polymer } \\
\hline PAL-NaPAA & $\mathrm{Si}$ & $5.0 \mathrm{~N} / \mathrm{cm}$ & $1914(100$ cycles, $0.84 \mathrm{~A} / \mathrm{g})$ & {$[17]$} \\
\hline Carboxymethyl fenugreek gum & $\mathrm{Si}$ & - & $1790(200$ cycles, $1 \mathrm{~A} / \mathrm{g})$ & {$[18]$} \\
\hline $\mathrm{CCS}$ & $\mathrm{S}$ & $\sim 4 \mathrm{~N}$ & $\sim 600(400$ cycles, $0.5 C)$ & [19] \\
\hline PF-COONa & $\begin{array}{l}\mathrm{Sn} \\
\mathrm{Si}\end{array}$ & $-\overline{6} \mathrm{~N}$ & $\begin{array}{c}518(500 \text { cycles, } 0.2 \mathrm{~A} / \mathrm{g}) \\
2806\left(100 \text { cycles, } 0.42 \mathrm{~mA} / \mathrm{g}, 0.19 \mathrm{mg} / \mathrm{cm}^{2}\right)\end{array}$ & [20-21] \\
\hline \multicolumn{5}{|c|}{ Cross-linked polymer } \\
\hline c-PEO-PEDOT:PSS/PEI & $\mathrm{Si}$ & $\sim 0.55 \mathrm{~N} / \mathrm{mm}^{2}$ & 2027 (500 cycles, 1.0 A/g) & {$[22]$} \\
\hline c-PAM-0.001 & $\mathrm{Si}$ & $13.98 \mathrm{~N}$ & 2834 (100 cycles, $0.1 C)$ & {$[23]$} \\
\hline PEI-ER & S & - & 1025 (500 cycles, $0.5 C)$ & {$[24]$} \\
\hline Cross-linked corn starch & $\mathrm{Si}$ & $31.2 \mathrm{gf} / \mathrm{cm}$ & 2106 (200 cycles, $0.5 C)$ & {$[25]$} \\
\hline SHP-PEG & $\mathrm{Si}$ & $\sim 3.2 \mathrm{~N} / \mathrm{cm}$ & $\sim 1300$ (150 cycles, $0.5 C)$ & {$[26]$} \\
\hline
\end{tabular}




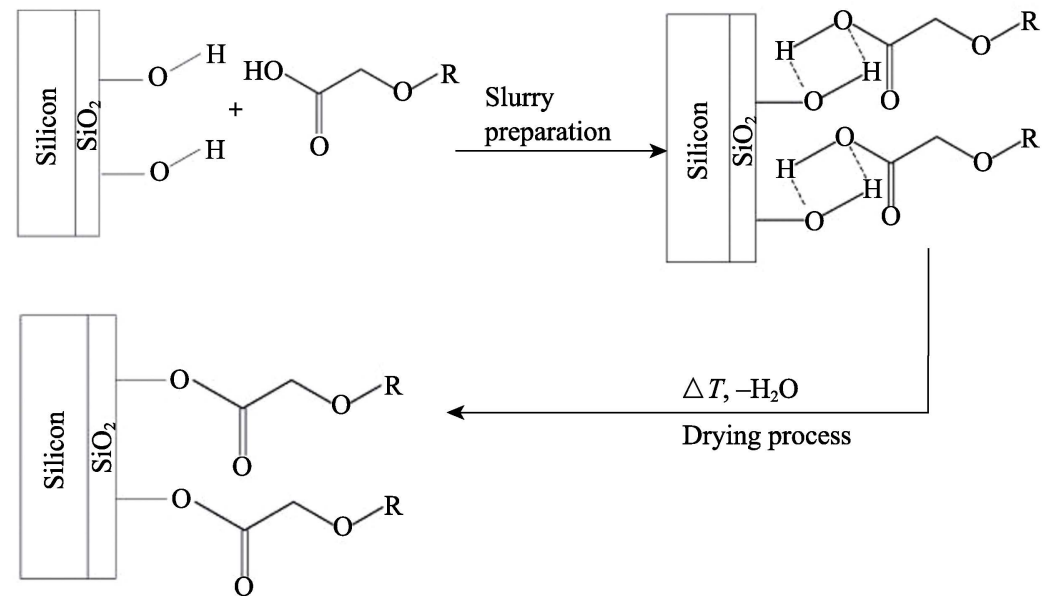

图 $2 \mathrm{CMC}$ 粘结剂对 $\mathrm{Si}$ 颗粒的粘结机理 ${ }^{[16]}$

Fig. 2 Mechanism of the CMC binder with Si nanoparticles ${ }^{[16]}$
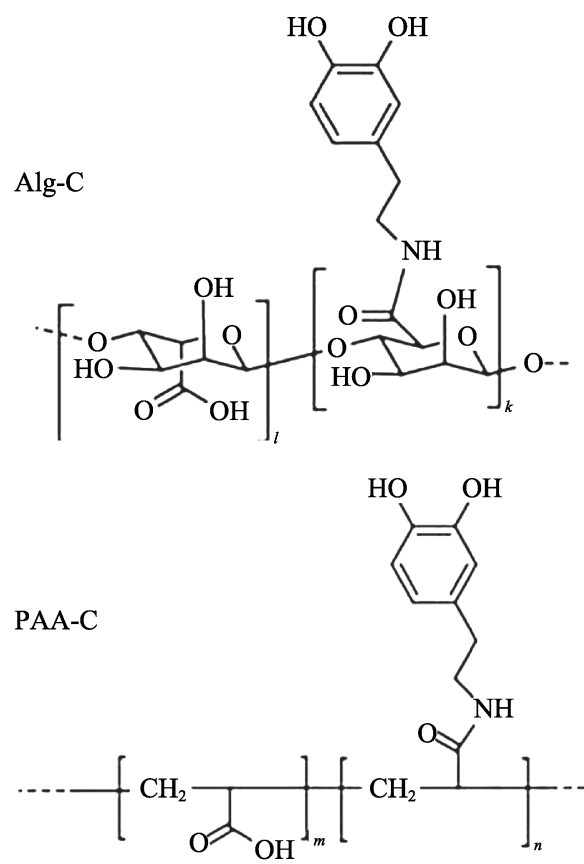

图 3 ALg-C 和 PAA-C 的化学结构式 ${ }^{[29]}$

Fig. 3 Molecular structures of ALg-C and PAA-c ${ }^{[29]}$

生的应力; 如果极性结构单元含量过小, 聚合物粘 结性差, 抗张强度低, 不利于抑制活性物质的体积 膨胀, 故需使极性聚合物中极性基团和非极性基团 结构单元配比合适, 如 $\mathrm{CMC}$ 和丁苯橡胶(SBR)混合 物。嵌锂过程中 $\mathrm{Si}$ 体积膨胀时, 由于 $\mathrm{CMC} / \mathrm{SBR}$ 中 SBR 的分子链易于运动, 有效防止了粘结剂的破裂 和活性物质的脱落, $\mathrm{CMC}$ 则提供较强粘结力和抗张 强度, 缓解 $\mathrm{Si}$ 体积膨胀, 所以 $\mathrm{CMC} / \mathrm{SBR}$ 粘结剂制 备的 $\mathrm{Si}$ 负极具有良好的电化学循环稳定性 ${ }^{[31]}$ 。

\section{2 链结构}

聚合物链结构也影响聚合物的物性。聚合物按 链结构形态分为线型结构和体型结构。线型结构聚合 物如 PAA、CMC 和 SA 对活性物质的粘结性好 ${ }^{[11,32-33]}$ 。
PAA 粘结剂制备的纳米 $\mathrm{Si}$ 负极在 $0.5 C$ 的条件下循环 100 圈, 每圈的平均比容量损失率不到 $0.2 \%{ }^{[32]}$ 。 $\mathrm{SA}$ 粘结剂制备的纳米 $\mathrm{Si}$ 负极, 在电流密度为 $4200 \mathrm{~mA} / \mathrm{g}$ 的条件下循环 100 圈, 比容量为 $1700 \sim 2000 \mathrm{mAh} / \mathrm{g}^{[33]}$ 。 但是线型结构的分子链之间易于滑动，受力后易发 生永久变形, 导致活性物质团聚, 降低极片的比容 量。而体型聚合物即交联聚合物分子链不易滑动, 变形后可以回复到原状, 因此交联聚合物具有更强 的粘结性。此类聚合物作为电极粘结剂时, 可有效 防止脱嵌锂过程中活性物质颗粒团聚, 缓解电极比 容量衰减 ${ }^{[34-36]}$ 。如以 $N, N^{\prime}$-亚甲基双丙烯酰胺为交联 剂制备的交联聚丙酰胺粘结剂, 可极大提高 $\mathrm{Si}$ 负极 的循环稳定性。在 $0.1 C$ 的条件下循环 100 圈后, 可 逆比容量达 $2843 \mathrm{mAh} / \mathrm{g}^{[23]}$ 。交联聚合物包括可逆交 联和不可逆交联聚合物, 可逆交联聚合物的交联点 具有可逆性。活性物质体积膨胀时破坏可逆交联点, 活性物质体积收缩时可逆交联点重新形成, 再次将 活性物质包裹在聚合物交联体中, 保证电极片的完

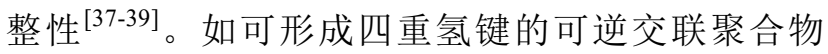
UPy-PAA 为 $\mathrm{Si}$ 负极的粘结剂时, 首次放电比容量高 达 $4194 \mathrm{mAh} / \mathrm{g}$, 循环 110 圈后可逆比容量仍然有 $2638 \mathrm{mAh} / \mathrm{g}^{[37]}$ 。Xu 等 ${ }^{[38]}$ 制备的可逆交联聚合物粘 结剂 PAA-P(HEA-co-DMA) 可提高 $\mathrm{Si}$ 负极的循环稳 定性。此粘结剂制备的 $\mathrm{Si}$ 负极循环 220 圈后比容量 仍有 $2394 \mathrm{mAh} / \mathrm{g}$ 。但是由于可逆交联点的作用力比 共价键弱, 对于体积膨胀巨大的活性物质, 聚合物 粘结剂中大部分交联点被破坏, 破坏了聚合物的体 型结构, 造成部分活性物质在体积收缩之前脱落, 降低了电极片的比容量。不可逆交联聚合物的不可 逆交联点通常为键能很高的化学键, 故不可逆交联 点一旦破坏便不可再次形成。如果交联聚合物中同 时存在可逆交联点和不可逆交联点, 则由于不可逆 
交联点的作用力高于可逆交联点，活性物质体积膨 胀产生的内应力优先使可逆交联点断裂并吸收能量, 有效防止了不可逆交联点的断裂, 可以保证聚合物 的体型结构, 防止活性物质脱落, 进而确保电极片 的完整性。鉴于此，可使粘结剂中同时存在不可逆 交联和可逆交联点，两者的协同作用进一步保证了 充放电过程中极片的完整性。此外, 聚合物的其他 结构特征也对电极的电化学性能影响很大。如具有 聚轮烷结构的粘结剂的断裂伸长率极高, 有效增加 了承受活性物质体积膨胀的能力，提高了电极的电 化学性能。Choi 等 ${ }^{[8]}$ 制备的含有聚轮烷的粘结剂 PR-PAA 的断裂伸长率可达 $390 \%$ 。此粘结剂可使 $\mathrm{Si}$ 负极在 $0.2 C$ 的条件下循环 150 圈后, 比容量为 $2.43 \mathrm{mAh} / \mathrm{cm}^{2}$, 容量保留率为 $91 \%$ 。

\section{2 极性聚合物的粘结性}

粘结性是粘结剂最重要的性能。高粘结性的粘 结剂可有效提高 LIBs 的电化学性能。极性聚合物粘 结剂的粘结性强弱不仅与聚合物分子链间作用力有 关，也与聚合物和被粘物质间相互作用力有关。上 述两种作用力主要为不可逆化学键和可逆的分子间 作用力如氢键和范德华力等。作用力较强的化学键 和氢键等有利于提高粘结剂的粘结性, 且作用力越 强, 粘结力越强。可逆作用力有利于保证极片的完 整性。如 $\mathrm{CMC}$ 与 $\mathrm{Si}$ 之间形成的共价键和氢键。嵌 锂过程中, 活性物质发生体积膨胀, 破坏了共价键 和氢键。共价键不能再次形成, 氢键可再次形成, 具 有自愈合能力(图 4), 有利于保证极片的完整性，提 高极片的电化学循环性能 ${ }^{[40]}$ 。但是具有可逆性的范 德华力作用力非常弱, 造成粘结力差, 不利于提高 电极的电化学稳定性, 特别是充放电过程中体积变 化巨大的 $\mathrm{Si} 、 \mathrm{Sn}$ 和 $\mathrm{S}$ 等活性物质。弱作用力无法缓 解和抑制充放电过程中活性物质的粉化和脱落。最
理想的状态是极性聚合物与活性物质间同时存在不 可逆化学键和作用力较强的可逆作用力。化学键提 供高粘结力, 可逆作用力吸附脱落和粉化的活性物 质，从而保证电化学循环过程中极片的完整性。

交联可进一步提高聚合物的粘结性能 ${ }^{[41]}$ 。如 $\mathrm{Ca}^{2+}$ 与 $\mathrm{SA}$ 制备的高度交联海藻酸钠粘结剂(图 5(a)) 可使 $\mathrm{Si}$ 极片的剥离强度远高于 PVDF 制备的 $\mathrm{Si}$ 极

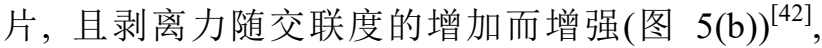
即交联度与极性聚合物的粘结性也有关。此外, $\mathrm{pH}$ 也会影响极性聚合物的粘结性。如 $\mathrm{pH}=3$ 的缓冲溶 液中, $\mathrm{CMC}$ 中的 $-\mathrm{COO}^{-}$和 $\mathrm{Si}$ 表面的 $\mathrm{SiO}^{-}$同时转化为 $-\mathrm{COOH}$ 和 $\mathrm{SiOH}$, 这两个基团易于形成酯键 ${ }^{[43]}$, 提 高粘结力。其他一些极性聚合物粘结剂的粘结性能 如表 1 所示。

极性聚合物的粘结性与极片的干燥条件和活性 物质的负载量有关。由于极片干燥时, 表面张力作 用使溶剂分子向极片表面运动，造成极片表面粘结 剂较多而靠近集流体部分粘结剂较少, 粘结力差。 升高温度时, 分子运动速度加快, 增加了粘结剂的 分布不均匀性, 造成粘结性降低 ${ }^{[44]}$ 。活性物质的负

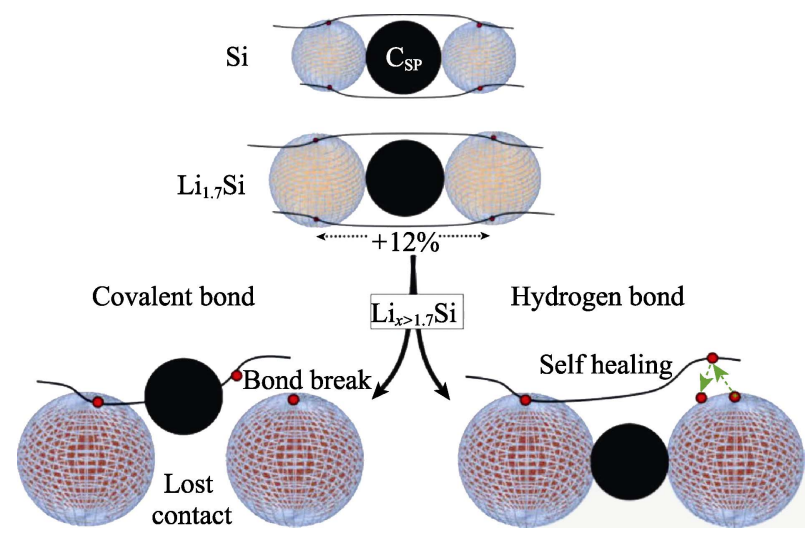

图 4 嵌锂时 $\mathrm{CMC}$ 与 $\mathrm{Si}$ 之间作用力的演变模型 $[40]$

Fig. 4 Model of the evolution in the CMC-Si bonds during lithiation process ${ }^{[40]}$

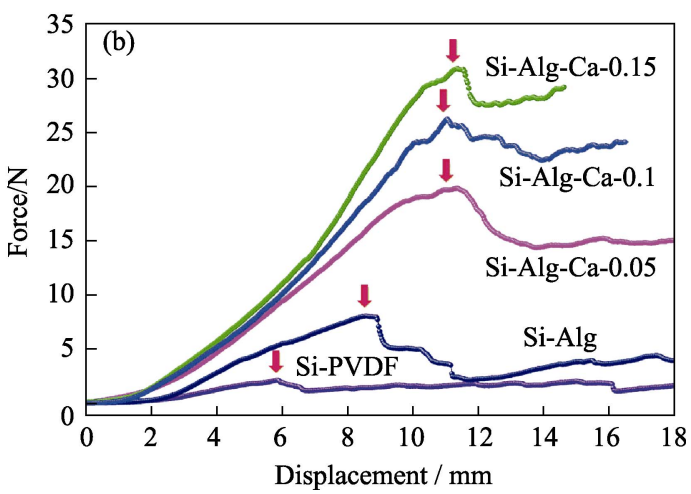

图 5 (a)高度交联 $\mathrm{SA}$ 粘结剂制备的 $\mathrm{Si}$ 极片的构建示意图, (b)不同粘结剂制备的 $\mathrm{Si}$ 负极的剥离强度 ${ }^{[42]}$

Fig. 5 (a) Architecture of Si electrode with highly cross-linked alginate binder, and (b) peeling forces of Si anodes with different binders ${ }^{[42]}$ 
载量增大，会增加极片的厚度，加剧粘结剂的分布 不均匀，也会降低粘结性 ${ }^{[45]}$ 。

故设计极性聚合物粘结剂时，需考虑 $\mathrm{pH}$ 、交联 剂和交联度等因素。在不同 $\mathrm{pH}$ 条件下极性基团的 活性有所区别, 所以选择合适的 $\mathrm{pH}$ 有利于提高粘 结性。交联剂不但对交联极性聚合物的粘结性能有 影响，而且从加工性能方面考虑，不同交联剂的交 联条件也有所不同，所以选择合适的交联剂至关重 要。交联剂用量对极性聚合物的交联度和粘结性能 都有影响。而选择适当的交联度, 可以提高聚合物 与被粘物质间的作用力。此外，调节极性基团的种 类和数量也有利于提高粘结剂的粘结性。

\section{3 极性聚合物的力学性能}

高比容量活性物质如 $\mathrm{Si}$ 在嵌锂过程中体积膨胀 可达 $300 \%$ 以上 ${ }^{[46]}$, 所以极性聚合物良好的弹性模 量、抗张强度、断裂伸长率、柔性和动态力学性能 等力学性能有利于抑制活性物质体积膨胀和适应活 性物质体积变化。高弹性模量和抗张强度的粘结剂有 利于抑制活性物质体积膨胀，防止活性物质粉化 ${ }^{[47]}$; 较高的断裂伸长率和良好的柔性有利于承受活性物 质的体积膨胀，防止活性物质脱落 ${ }^{[23]}$ 。充放电循环 过程中活性物质体积变化时, 优异的动态力学性能
可有效防止极性聚合物破裂，确保极片的完整性。 因为聚合物分子内化学键与分子间作用力可以抵抗 外力的破坏，所以增加极性聚合物的极性基团数 量、增强分子间作用力和提高分子量等均可改善聚 合物的弹性模量和抗张强度 ${ }^{[32,48-49]}$, 更有利于抑制 活性物质体积膨胀。但是过大的分子间作用力，降 低了分子链的运动能力, 极大地影响了聚合物的断 裂伸长率和柔性。交联可提高聚合物的弹性模量和 抗张强度, 降低聚合物的断裂伸长率。如以 $N$-差弪甲 基丙烯酰胺 (PNA) 为交联剂制备的粘结剂 (PNA-NaPAA-g-CMC, 图 6(a)) 的抗张强度远高于其 他线型聚合物，但是断裂伸长率非常小(图 6(b))。该 粘结剂可提高 $\mathrm{Si}$ 电极的电化学循环稳定性能, 循环 250 圈后比容量仍然在 $2000 \mathrm{mAh} / \mathrm{g}$ 以上(图 6(c) $)^{[47]}$ 。 此外，增大分子量和提高结晶度均可提高聚合物的 弹性模量和抗张强度 ${ }^{[50]}$ 。故可通过调节聚合物极性 基团的种类和数量、分子间作用力、交联度、结晶 度等来提高聚合物机械性能。

\section{4 极性聚合物的导电性}

传统负极活性物质石墨具有良好的导电性, 但 是高比容量活性物质 $(\mathrm{Si} 、 \mathrm{Sn}$ 和 $\mathrm{S}$ 等)的导电性差, 需 添加导电剂提高电极的导电性。尽管通常所用的炭

(a)

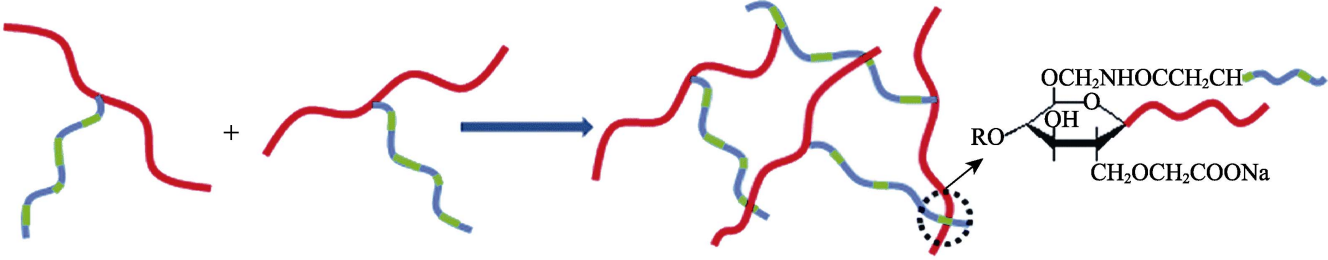

AA: $\mathrm{CH}_{2}=\mathrm{CH}-\mathrm{COOH} \quad$ NA: $\mathrm{CH}_{2}=\mathrm{CH}-\mathrm{COOH}-\mathrm{CH}_{2} \mathrm{OH}$

$\sim: \mathrm{CMC}$

: PAA-Na

: PAN
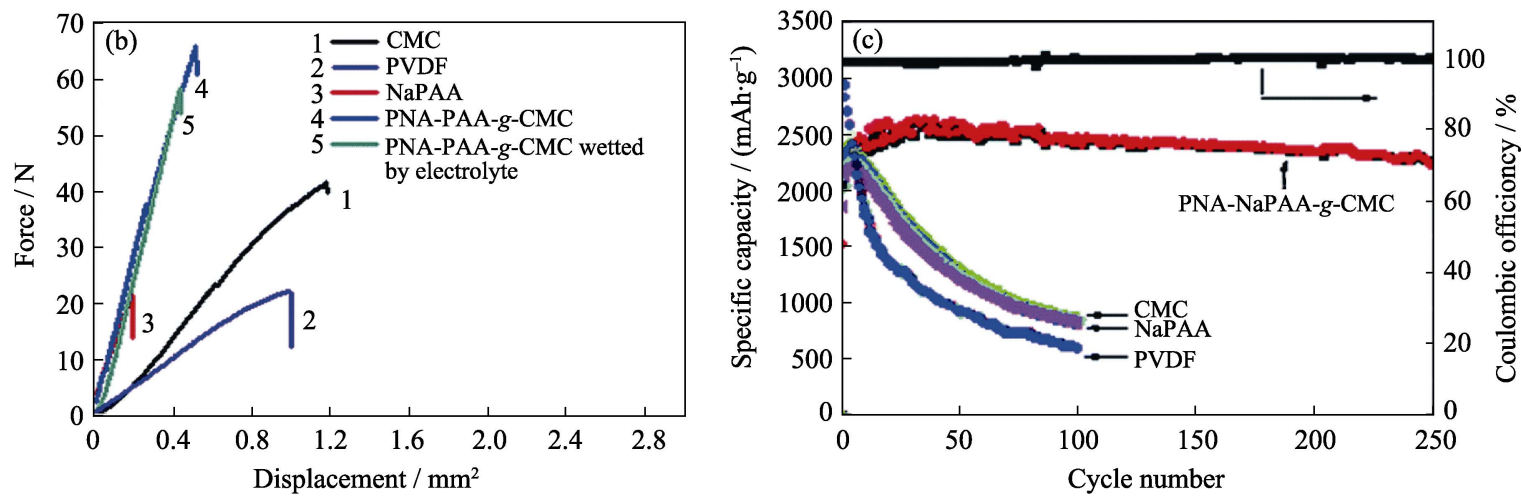

图 6 (a)交联粘结剂 PNA-NaPAA-g-CMC 的示意图, (b)电解质浸泡后的不同粘结剂的拉伸曲线和 (c)不同粘结剂制备的 $\mathrm{Si}$ 电极的循环曲线 ${ }^{[47]}$

Fig. 6 (a) Scheme for the cross-linked binder of PNA-NaPAA- $g$-CMC, (b) tensile tests of different binders impregnated with electrolyte and (c) cycling performances of Si electrodes with different binders ${ }^{[47]}$ 
黑等无机材料导电剂可提高电极的导电性, 但是 其在充放电过程中易团聚或脱落, 破坏电极的导

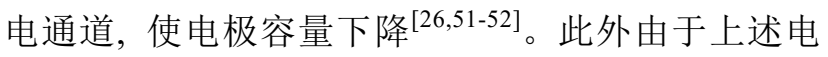
极的 $\mathrm{Li}^{+}$传输能力差, 不利于 LIBs 的倍率性能。使 用有利于电子传输和(或)离子传输的导电聚合物 粘结剂可减少无机导电剂的添加量, 提高 LIBs 的 倍率性能 ${ }^{[20,53]}$ 。

导电聚合物分为复合型和结构型导电聚合物, 这里主要论述结构型导电极性聚合物。结构型导电 极性聚合物又分为电子传输型(聚吡咯 ${ }^{[54]}$ 、聚苯胺 ${ }^{[55]}$ 和酞菁铜(II)四磺酸盐交联的聚吡咯 ${ }^{[56]}$ )和离子传输 型导电聚合物(如聚丙烯酸锂 ${ }^{[57]}$ 、瓜尔豆胶 ${ }^{[58]}$ )。同 时具有良好的电子和离子传输性的导电极性聚合物 可同时提高电极的电子和离子传输速率，故结合两 种聚合物或将有利于电子和离子传输的基团同时引 入聚合物链中, 如聚氧化乙烯、聚乙烯亚胺和聚 (3,4-亚乙二氧基噻吩)-聚(苯乙烯磺酸)的复合物 (c-PEO-PEDOT:PSS/PEI) 的离子传输速率和电子传 输速率都远高于乙炔黑掺杂 $\mathrm{CMC}$, 分别是其 14 倍 和 90 倍。c-PEO-PEDOT:PSS/PEI 为导电粘结剂制 备的 $\mathrm{Si}$ 电极在 $8.0 \mathrm{~A} / \mathrm{g}$ 的高电流密度下比容量可达 $1500 \mathrm{mAh} / \mathrm{g}^{[22]}$ 。

\section{5 极性聚合物的其他性能}

粘结剂的其他性能如吸液率、化学和电化学稳 定性以及是否易于形成稳定的固体电解质界面(SEI) 膜等均影响 LIBs 的电化学性能。

粘结剂吸液率会影响 $\mathrm{Li}^{+}$的传输速率和粘结性 能。高吸液率有利于 $\mathrm{Li}^{+}$传输 ${ }^{[21]}$, 但由于增加了极性 聚合物与被粘物质界面上电解液的量, 降低了粘结 剂的粘结性能 ${ }^{[59-60]}$, 故极性聚合物需具有适量的吸 液率。但是由极性聚合物中的微孔造成的高吸液率 则不影响粘结剂的粘结性能。所以为了使粘结剂同 时具有优异的吸液率和粘结性, 极性聚合物中引入 可包裹电解液的特殊结构, 可提高 $\mathrm{Li}^{+}$的传输速率, 减少电解液与活性物质的直接接触。如以 PAA 为交 联剂制备的交联型阿拉伯树胶 $(\mathrm{GA}-\mathrm{PAA})^{[61]}$ 的吸液 率可达 $25 \mathrm{wt} \%$, 高于 PAA 的 $\mathrm{Li}^{+}$传输速率。本课题 组由 PAA 和乙烯一醋酸乙烯酯共聚物(EVA)制备的 $\mathrm{PAA} / \mathrm{EVA}$ 粘结剂可将电解液包裹在 EVA 颗粒中(图 7)。 PAA/EVA 电极片的吸液率高达 $59 \%$, 而 PAA 电极 片的吸液率仅为 $15 \%$ 。此粘结剂的高吸液率对粘结 性能影响很小 ${ }^{[62]}$ 。LIBs 用极性聚合物粘结剂还需具 有化学和电化学稳定性。极性聚合物粘结剂的化学 稳定性是指其不与活性物质、导电剂以及电解质

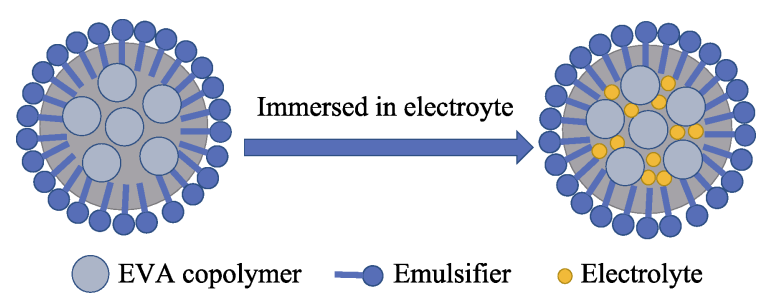

图 7 EVA 颗粒在电解液中浸泡的结构示意图 ${ }^{[62]}$

Fig. 7 Schematic for the process of EVA colloid immersed in electrolyte ${ }^{[62]}$

发生反应的能力，与其分子结构和化学环境有关。 PVDF 是一种非常稳定的聚合物, 但是在由碳酸乙 烯酯、碳酸二乙酯和碳酸二甲酯组成的有机溶剂 中易发生溶胀 ${ }^{[63]}$ 。极性聚合物粘结剂的电化学稳 定性是指粘结剂在 LIBs 的工作电压下不发生氧 化还原反应的能力。聚合物粘结剂的电化学稳定 性与粘结剂的活化能有关, 其电化学反应符合能 斯特方程 ${ }^{[64]}$ 。

SEI 膜可防止电解质和 $\mathrm{Li}^{+}$的进一步消耗，也可 阻止溶剂分子共嵌入，保护电极材料。但是 SEI 膜 的形成同时消耗了 $\mathrm{Li}^{+}$和电解液, 电极的不可逆容 量增加, 因此薄且稳定的 SEI 膜对提高 LIBs 可逆容 量的循环稳定性和倍率等性能至关重要。粘结剂对 形成稳定的 SEI 膜有重要影响。极性聚合物因含有 $-\mathrm{COOH} 、-\mathrm{OH}$ 和 $-\mathrm{NH}^{2}$ 等极性基团, 可与表面含极 性基团的活性物质形成化学键或氢键, 减少电解质 与活性物质的接触, 进而减小 SEI 膜的厚度 ${ }^{[18,65]}$ 。

传统粘结剂 PVDF 对环境污染严重, 不利于操 作人员的健康, 故粘结剂的环保和安全性也是需考 虑的重要因素。目前已有很多学者致力于开发环保、 安全的水性粘结剂, 如壳聚糖及其衍生物 ${ }^{[6-67]}$ 、聚乙 烯亚胺及其衍生物 ${ }^{[5,68-69]}$ 、聚丙烯酸及其衍生物 ${ }^{[70-72]}$ 等, 但是还需要进一步提高 LIBs 的比容量、首次库 伦效率和循环稳定性等。

\section{6 总结与展望}

极性聚合物作为 LIBs 的主要粘结剂, 对 LIBs 性能的影响主要与聚合物的结构和物性有关, 如聚 合物的结构特征、粘结性、力学性能、导电性等。 结构特征主要与聚合物的化学结构有关, 分子链中 引入适量可生成可逆或(和)不可逆交联点的特定极 性基团, 形成具有三维网络结构的极性聚合物, 可 提高极性聚合物的弹性模量、抗张强度、断裂伸长 率和柔性, 保证充放电过程中极片的完整性。分子 链中引入具有良好电子和离子导电性的基团，可提 高电极的电化学循环性能和倍率性能。此外, 与活 
性物质表面形成化学键或其他分子间作用力(如氢 键) 的极性聚合物有利于形成薄且稳定的 SEI 膜。目 前已探索研究出了一些具有优异性能的粘结剂, 但 还不能同时满足上述所有要求, 特别是对于高比容 量的活性物质, 还存在制备复杂、不能完全抑制或 承受活性物质的体积膨胀等缺点, 造成电极的循环 稳定性和倍率性能差。未来需要设计合成或发现同 时具有优异粘结性、力学性能和导电性的新型粘结 剂, 进一步提高电极的电化学性能。对于极性聚合 物粘结剂的设计, 需通过调节极性单元的种类和数 量、聚合物分子量、交联剂种类和用量、 $\mathrm{pH}$ 、有利 于电子和离子导电性的结构等提高粘结剂的性能。 此外, 目前适用于高低温环境的锂离子电池粘结剂 较少, 需进一步研究开发。

\section{参考文献:}

[1] HE W, TIAN H, ZHANG S, et al. Scalable synthesis of Si/C anode enhanced by $\mathrm{FeSi}_{x}$ nanoparticles from low-cost ferrosilicon for lithium-ion batteries. J. Power Sources, 2017, 353: 270-276.

[2] CHEN Y, LIU L, XIONG J, et al. Porous Si nanowires from heap metallurgical silicon stabilized by a surface oxide layer for lithium ion batteries. Adv. Funct. Mater., 2015, 25(43): 6701-6709.

[3] PARK M H, KIM M G, JOO J, et al. Silicon nanotube battery anodes. Nano Lett., 2009, 9(11): 3844-3847.

[4] LIU Y, TAI Z, ZHOU T, et al. An all-integrated anode via interlinked chemical bonding between double-shelled-yolkstructured silicon and binder for lithium-ion batteries. Adv. Mater., 2017, 29(44): 1703028-1-11.

[5] AKHTAR N, SHAO H, AI F, et al. Gelatin-polyethylenimine composite as a functional binder for highly stable lithium-sulfur batteries. Electrochim. Acta, 2018, 282: 758-766.

[6] HE J, ZHANG L. Polyvinyl alcohol grafted poly (acrylic acid) as water-soluble binder with enhanced adhesion capability and electrochemical performances for Si anode. J. Alloy. Compound, 2018, 763: 228-240

[7] LING M, ZHANG L, ZHENG T, et al. Nucleophilic substitution between polysulfides and binders unexpectedly stabilizing lithium sulfur battery. Nano Energy, 2017, 38: 82-90.

[8] CHOI S, KWON T W, COSKUN A, et al. Highly elastic binders integrating polyrotaxanes for silicon microparticle anodes in lithium ion batteries. Science, 2017, 357(6348): 279-283.

[9] ZHAO X Y, YIM C H, DU N Y, et al. Crosslinked chitosan networks as binders for silicon/graphite composite electrodes in Li-ion batteries. J. Electrochem. Soc., 2018, 165(5): A1110-A1121.

[10] HU S, CAI Z, HUANG T, et al. A modified natural polysaccharide as a high-performance binder for silicon anodes in lithium-ion batteries. ACS Appl. Mater. Interfaces, 2019, 11(4): 4311-4317.

[11] DROFENIK J, GABERSCEK M, DOMINKO R, et al. Cellulose as a binding material in graphitic anodes for $\mathrm{Li}$ ion batteries: a performance and degradation study. Electrochim. Acta, 2003, 48(7): 883-889.

[12] ZHANG R, YANG X, ZHANG D, et al. Water soluble styrene butadiene rubber and sodium carboxyl methyl cellulose binder for
$\mathrm{ZnFe}_{2} \mathrm{O}_{4}$ anode electrodes in lithium ion batteries. J. Power Sources, 2015, 285: 227-234.

[13] MIN K, RAMMOHAN A R, LEE S H, et al. Grafting functional groups in polymeric binder toward enhancing structural integrity of $\mathrm{Li}_{x} \mathrm{SiO}_{2}$ anode during electrochemical cycling. J. Phys. Chem. C, 2018, 122(30): 17190-17198.

[14] YOO J K, JEON J, KANG K, et al. Glyoxalated polyacrylamide as a covalently attachable and rapidly cross-linkable binder for $\mathrm{Si}$ electrode in lithium ion batteries. Electron. Mater. Lett., 2017, 13(2): 136-141.

[15] JIAO Y, CHEN W, LEI T, et al. A Novel polar copolymer design as a multi-functional binder for strong affinity of polysulfides in lithium-sulfur batteries. Nanoscale Res. Lett., 2017, 12: 195-1-8.

[16] HOCHGATTERER N S, SCHWEIGER M R, KOLLER S, et al. Silicon/graphite composite electrodes for high-capacity anodes: influence of binder chemistry on cycling stability. Electrochem. Solid State Lett., 2008, 11(5): A76-A80.

[17] LUO C, DU L, WU W, et al. Novel lignin-derived water-soluble binder for micro silicon anode in lithium-ion batteries. ACS Sustain. Chem. Eng., 2018, 6(10): 12621-12629.

[18] QIU L W, SHEN Y D, FAN H B, et al. Carboxymethyl fenugreek gum: rheological characterization and as a novel binder for silicon anode of lithium-ion batteries. Int. J. Biol. Macromol., 2018, 115: 672-679.

[19] YI H, LAN T, YANG Y, et al. Aqueous-processable polymer binder with strong mechanical and polysulfide-trapping properties for high performance of lithium-sulfur batteries. J. Mater. Chem. A, 2018, 6 (38): 18660-18668.

[20] ZHAO Y, YANG L Y, LIU D, et al. A conductive binder for high-performance Sn electrodes in lithium-ion batteries. ACS Appl. Mater. Interfaces, 2018, 10(2): 1672-1677.

[21] LIU D, ZHAO Y, TAN R, et al. Novel conductive binder for high-performance silicon anodes in lithium ion batteries. Nano Energy, 2017, 36: 206-212.

[22] ZENG W, WANG L, PENG X, et al. Enhanced ion conductivity in conducting polymer binder for high-performance silicon anodes in advanced lithium-ion batteries. Adv. Energy Mater., 2018, 8(11): 1702314-1-8

[23] ZHU X, ZHANG F, ZHANG L, et al. A highly stretchable cross-linked polyacrylamide hydrogel as an effective binder for silicon and sulfur electrodes toward durable lithium-ion storage. Adv. Funct. Mater., 2018, 28(11): 1705015-1-12.

[24] YAN L, GAO X, WAHID-PEDRO F, et al. A novel epoxy resin-based cathode binder for low cost, long cycling life, and high-energy lithium-sulfur batteries. J. Mater. Chem. A, 2018, 6(29): 14315-14323.

[25] ROHAN R, KUO T C, CHIOU C Y, et al. Low-cost and sustainable corn starch as a high-performance aqueous binder in silicon anodes via in situ cross-linking. J. Power Sources, 2018, 396: 459-466.

[26] MUNAOKA T, YAN X Z, LOPEZ J, et al. Ionically conductive self-healing binder for low cost $\mathrm{Si}$ microparticles anodes in Li-ion batteries. Adv. Energy Mater., 2018, 8(14): 1703138-1-11.

[27] LUO L, XU Y, ZHANG H, et al. Comprehensive understanding of high polar polyacrylonitrile as an effective binder for Li-ion battery nano-si anodes. ACS Applied Materials \& Interfaces, 2016, 8(12): 8154-8161.

[28] PARK H K, KONG B S, OH E S. Effect of high adhesive polyvinyl alcohol binder on the anodes of lithium ion batteries. Electro- 
chem. Commun., 2011, 13(10): 1051-1053.

[29] RYOU M H, KIM J, LEE I, et al. Mussel-inspired adhesive binders for high-performance silicon nanoparticle anodes in lithium-ion batteries. Adv. Mater., 2013, 25(11): 1571-1576.

[30] WEI L, CHEN C, HOU Z, et al. Poly (acrylic acid sodium) grafted carboxymethyl cellulose as a high performance polymer binder for silicon anode in lithium ion batteries. Sci. Rep., 2016, 6: 19583-1-8.

[31] BUQA H, HOLZAPFEL M, KRUMEICH F, et al. Study of styrene butadiene rubber and sodium methyl cellulose as binder for negative electrodes in lithium-ion batteries. J. Power Sources, 2006, 161(1): 617-622.

[32] MAGASINSKI A, ZDYRKO B, KOVALENKO I, et al. Toward efficient binders for Li-ion battery Si-based anodes: polyacrylic acid. ACS Appl. Mater. Interfaces, 2010, 2(11): 3004-3010.

[33] KOVALENKO I, ZDYRKO B, MAGASINSKI A, et al. A major constituent of brown algae for use in high-capacity Li-ion batteries. Science, 2011, 334(6052): 75-79.

[34] HAN Z J, YABUUCHI N, HASHIMOTO S, et al. Cross-linked poly(acrylic acid) with polycarbodiimide as advanced binder for $\mathrm{Si} /$ graphite composite negative electrodes in Li-ion batteries. ECS Electrochem. Lett., 2013, 2(2): A17-A20.

[35] WANG L, LIU T, PENG X, et al. Highly stretchable conductive glue for high-performance silicon anodes in advanced lithium-ion batteries. Adv. Funct. Mater., 2017, 28(3): 1704858-1-8.

[36] YANG J, ZHANG L, ZHANG T, et al. Self-healing strategy for Si nanoparticles towards practical application as anode materials for Li-ion batteries. Electrochemistry Communications, 2018, 87: 22-26.

[37] ZHANG G, YANG Y, CHEN Y, et al. A wuadruple-hydrogenbonded supramolecular binder for high-performance silicon anodes in lithium-ion batteries. Small, 2018, 14(29): 1801189-1-10.

[38] XU Z, YANG J, ZHANG T, et al. Silicon microparticle anodes with self-healing multiple network binder. Joule, 2018, 2(5): $818-819$.

[39] KWON T W, JEONG Y K, DENIZ E, et al. Dynamic cross-linking of polymeric binders based on host-guest interactions for silicon anodes in lithium ion batteries. ACS Nano, 2015, 9(11): 11317- 11324.

[40] BRIDEL J S, AZAIS T, MORCRETTE $\mathrm{M}$, et al. In situ observation and long-term reactivity of $\mathrm{Si} / \mathrm{C} / \mathrm{CMC}$ composites electrodes for Li-ion batteries. J. Electrochem. Soc., 2011, 158(6): A750-A759.

[41] WEI Y J, WANG Z J, YE H, et al. A stable cross-linked binder network for $\mathrm{SnO}_{2}$ anode with enhanced sodium-ion storage performance. ChemistrySelect, 2017, 2(35): 11365-11369.

[42] ZHANG L, ZHANG L, CHAI L, et al. A coordinatively cross-linked polymeric network as a functional binder for high-performance silicon submicro-particle anodes in lithium-ion batteries. J. Mater. Chem. A, 2014, 2(44): 19036-19045.

[43] MAZOUZI D, LESTRIEZ B, ROUE L, et al. Silicon composite electrode with high capacity and long cycle life. Electrochemical and Solid-State Letters, 2009, 12(11): A215-A218.

[44] BAUNACH M, JAISER S, SCHMELZLE S, et al. Delamination behavior of lithium-ion battery anodes: influence of drying temperature during electrode processing. Dry. Techn., 2016, 34(4): 462-473.

[45] KARKAR Z, MAZOUZI D, HERNANDEZ C R, et al. Threshold-like dependence of silicon-based electrode performance on active mass loading and nature of carbon conductive additive. Electrochim. Acta, 2016, 215: 276-288.

[46] HE W, TIAN H, XIN F, et al. Scalable fabrication of micro-sized bulk porous Si from Fe-Si alloy as a high performance anode for lithium-ion batteries. J. Mater. Chem. A, 2015, 3(35): 17956- 17962.

[47] WEI L M, HOU Z Y. High performance polymer binders inspired by chemical finishing of textiles for silicon anodes in lithium ion batteries. J. Mater. Chem. A, 2017, 5(42): 22156-22162.

[48] NUNES R W, MARTIN J R, JOHNSON J F. Influence of molecularweight and molecular-weight distribution on mechanical-properties. Polym. Eng. Sci., 1982, 22(4): 205-228.

[49] WU Q, HENRIKSSON M, LIU X, et al. A high strength nanocomposite based on microcrystalline cellulose and polyurethane. Biomacromolecules, 2007, 8(12): 3687-3692.

[50] WANG Y, GOZEN A, CHEN L, et al. Gum-like nanocomposites as conformable, conductive, and adhesive electrode matrix for energy storage devices. Adv. Energy Mater., 2017, 7(6): 1601767-1-11.

[51] YUCA N, CETINTASOGLU M E, DOGDU M F, et al. Highly efficient poly(fluorene phenylene) copolymer as a new class of binder for high-capacity silicon anode in lithium-ion batteries. Int. J. Energy Res., 2018, 42(3): 1148-1157.

[52] HUANG S, REN J G, LIU R, et al. Low addition amount of self-healing ionomer binder for $\mathrm{Si} /$ graphite electrodes with enhanced cycling. New J. Chem., 2018, 42(9): 6742-6749.

[53] WANG Z Q, TIAN S K, LI S D, et al. Lithium sulfonate-grafted poly(vinylidenefluoride-hexafluoro propylene) ionomer as binder for lithium-ion batteries. RSC Adv., 2018, 8(36): 20025-20031.

[54] MILROY C, MANTHIRAM A. An elastic, conductive, electroactive nanocomposite binder for flexible sulfur cathodes in lithium-sulfur batteries. Adv. Mater., 2016, 28(44): 9744-9751.

[55] TAMURA T, AOKI Y, OHSAWA T, et al. Polyaniline as a functional binder for $\mathrm{LiFePO}_{4}$ cathodes in lithium batteries. Chem. Lett., 2011, 40(8): 828-830.

[56] SHI Y, ZHANG J, BRUCK A M, et al. A tunable 3D nanostructured conductive gel framework electrode for highperformance lithium ion batteries. Adv. Mater., 2017, 29(22): 1603922-1-8.

[57] PIECZONKA N P W, BORGEL V, ZIV B, et al. Lithium polyacrylate (LiPAA) as an advanced binder and a passivating agent for high-voltage Li-ion batteries. Adv. Energy Mater., 2015, 5(23): $1501008-1-10$.

[58] LIU J, ZHANG Q, ZHANG T, et al. A robust ion-conductive biopolymer as a binder for $\mathrm{Si}$ anodes of lithium-ion batteries. Adv. Funct. Mater., 2015, 25(23): 3599-3605.

[59] GENDENSUREN B, OH E S. Dual-crosslinked network binder of alginate with polyacrylamide for silicon/graphite anodes of lithium ion battery. J. Power Sources, 2018, 384: 379-386.

[60] PAN J, XU G, DING B, et al. PAA/PEDOT:PSS as a multifunctional, water-soluble binder to improve the capacity and stability of lithium-sulfur batteries. RSC Adv., 2016, 6(47): 40650-40655.

[61] LING M, ZHAO H, XIAO X, et al. Low cost and environmentally benign crack-blocking structures for long life and high power $\mathrm{Si}$ electrodes in lithium ion batteries. J. Mater. Chem. A, 2015, 3(5): 2036-2042.

[62] GUO R, ZHANG S, YING H, et al. A new, effective and low cost dual-functional binder for porous silicon anodes in lithium-ion batteries. ACS Appl. Mater. Interfaces, 2019, 11(15): 14051- 14058.

[63] CHEN Z, CHRISTENSEN L, DAHN J R. Comparison of PVDF and PVDF-TFE-P as binders for electrode materials showing large volume changes in lithium-ion batteries. J. Electrochem. Soc., 2003, 150(8): A1073-A1078. 
[64] LAIDLER K J. The development of the Arrhenius equation. $J$. Chem. Educ., 1984, 61(6): 494-498.

[65] KARKAR Z, GUYOMARD D, ROUE L, et al. A comparative study of polyacrylic acid (PAA) and carboxymethyl cellulose (CMC) binders for Si-based electrodes. Electrochim. Acta, 2017, 258: $453-466$.

[66] LEE S H, LEE J H, NAM D H, et al. Epoxidized natural rubber/chitosan network binder for silicon anode in lithium-ion battery. ACS Appl. Mater. Interfaces, 2018, 10(19): 16449-16457.

[67] GAO H, ZHOU W, JANG J H, et al. Cross-linked chitosan as a polymer network binder for an antimony anode in sodium-ion batteries. Adv. Energy Mater., 2016, 6(6): 1502130-1-7.

[68] WANG H, LING M, BAI Y, et al. Cationic polymer binder inhibit shuttle effects through electrostatic confinement in lithium sulfur batteries. J. Mater. Chem. A, 2018, 6(16): 6959-6966.
[69] LIAO J, LIU Z, LIU X, et al. Water-soluble linear poly (ethylenimine) as a superior bifunctional binder for lithium-sulfur batteries of improved cell performance. J. Phys. Chem. C, 2018, 122(45): 25917-25929.

[70] SONG J, ZHOU M, YI R, et al. Interpenetrated gel polymer binder for high-performance silicon anodes in lithium-ion batteries. Adv. Funct. Mater., 2014, 24(37): 5904-5910.

[71] FEI J, SUN Q Q, CUI Y L, et al. Sodium carboxyl methyl cellulose and polyacrylic acid binder with enhanced electrochemical properties for $\mathrm{ZnMoO}_{4} \cdot 0.8 \mathrm{H}_{2} \mathrm{O}$ anode in lithium ion batteries. $J$. Electroanal. Chem., 2017, 804: 158-164.

[72] LEE S Y, CHOI Y, HONG K S, et al. Influence of EDTA in poly(acrylic acid) binder for enhancing electrochemical performance and thermal stability of silicon anode. Appl. Surf. Sci., 2018, 447: $442-451$. 